\title{
Materials Data for Modeling, Simulation, and Analysis
}

\author{
Scott D. Henry
}

Published online: 28 August 2012

(C) Springer Science+Business Media, LLC and ASM International 2012

The materials science and engineering world has been focusing much attention in recent months on issues related to materials data, and the innovations and advancements needed to speed up the development of new materials and processing methods. In June 2011, the Materials Genome Initiative (MGI) was announced by the National Science and Technology Council of the Executive Office of the President. The MGI is a "new, multi-stakeholder effort to develop an infrastructure to accelerate advanced materials discovery and deployment in the United States." The ultimate goal of MGI is to fast-track the development and deployment of materials for critical medical, transportation, and national security applications. Similar programs have been announced in the European Union, and it is fair to say that research and development groups across the globe are looking for ways to speed up materials innovations.

In May 2012, the US National Institute of Standards and Technology (NIST) held a workshop in Washington, DC, "Building the Materials Innovation Infrastructure: Data and Standards." Participants represented a broad cross section of materials research and development organizations, with experience in materials at various length scales (from nano to macro) and application areas. Some of the most pressing issues identified by participants were related to

- Finding and accessing legacy data.

- Archiving data.

S. D. Henry $(\bowtie)$

ASM International, 9639 Kinsman Road,

Materials Park, OH 44073, USA

e-mail: Scott.henry@asminternational.org

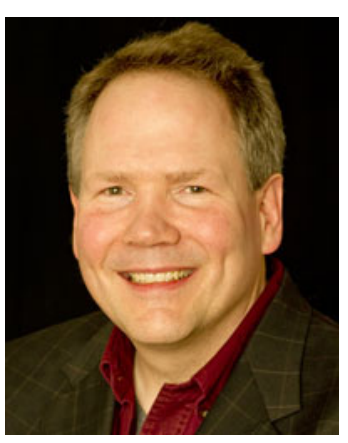

- Lack of standards and a common schema for data capture and sharing.

- Ownership, intellectual property, and security issues that prevent sharing and collaboration.

At a White House event scheduled in conjunction with the workshop, ASM International announced the launch of a national Center for Computational Materials Science and Engineering Data (CCMSED); the name of the center may change as its work evolves. The goal of the CCMSED is to serve as the leading organization for the collection, dissemination, and management of materials data. The center will support advanced computational materials science and engineering by completing projects that enable specific materials and processing "ecosystems" to develop, share, and manage large amounts of data for the advancement of research and product development goals and the rapid development of next generation materials.

The chair of the ASM team leading the effort is David Furrer, FASM, senior fellow discipline lead, Pratt \& Whitney. The center will be a collaborative effort to develop next generation data structures, tools, and data exchange systems. The focus on critical "ecosystems" will 
help advance this effort through addressing real-world problems.

Two ecosystems have been selected for initial projects: Aerospace Structural Materials Data and Manufacturing In-Process Materials Data. Project teams are being organized, including representation from key organizations and industry groups.

The aerospace materials group is being developed in partnership with the Materials Data Management Consortium (MDMC), a group of materials-oriented organizations in the energy, aerospace, and defense sectors. The MDMC has 10 years of experience in developing enterprise materials data management solutions.

The manufacturing data team plans to focus on in-process, high-temperature data such as yield stress and flow stress and their use in process modeling and supply chain data transfer. The technical roadmaps developed by groups such as the ASM Heat Treating Society emphasize the growing importance of modeling and the need for highquality data to develop, validate, and refine models. The work of the CCMSED will help expand the use and increase the power of process modeling.

Further ecosystem areas are being explored in collaboration with The Minerals, Metals \& Materials Society (TMS).

CCMSED will develop teams of key stakeholders to define protocols, establish data management infrastructure, and assist in the development of national standards to support internal organizational and supply-chain needs. The center will support NIST, which was charged with leading the federal government's efforts in developing materials data standards.

As part of its mission, CCMSED will define, create, and demonstrate:

- Database structures, import and export interface standards, data format and quality requirements for rapid materials innovation and development.

- Data sharing and collaboration across stakeholder organizations, including industry, academia, and government.

- Collection, maintenance, and dissemination of data unique to specific materials and processing sectors, such as dynamic properties associated with in-process manufacturing of materials.

- Data integration with software for multiscale modeling and model validation/verification and data visualization.

- Data collection and processing to create a large-scale database(s) of highly pedigreed materials data, both experimental and calculated.

- An array of software tools for exploring, visualizing, and analyzing the data; for materials selection, substitution, and design; and for export to and interface with engineering analysis tools.

- A comprehensive online index of other materials databases, with information about source, scope, data pedigree, and accessibility.

ASM International is seeking feedback and participation from materials professionals and groups may be interested in participating or collaborating in this effort. 